\title{
The Impact of PPARD and PPARG Polymorphisms on Glioma Risk and Prognosis
}

\author{
Xiaoying Ding ${ }^{1}$, Xinsheng $\mathrm{Han}^{1}$, Haozheng Yuan ${ }^{1}$, Yong Zhang ${ }^{1} \& \mathrm{Ya} \mathrm{Gao}^{2 *}$
}

Recent studies showed that peroxisome proliferator-activated receptors (PPARs) had effects on the progression of multiple tumors, but the role of PPARD and PPARG in glioma remains poorly understand. We conducted a case-control study to investigate the association of polymorphisms in PPARD and PPARG with glioma risk and prognosis in the Chinese Han population. Seven polymorphisms (PPARD: rs2016520, rs67056409, rs1053049 and rs2206030; PPARG: rs2920503, rs4073770 and rs1151988) were genotyped using the Agena MassARRAY system in 568 glioma patients and 509 healthy controls. The odd ratios (OR) and $95 \%$ confidence interval $(\mathrm{Cl})$ were calculated to assess the association of PPARD and PPARG polymorphisms with glioma risk. The Multifactor dimensionality reduction (MDR) method was used to analysis interactions of genetic polymorphisms on glioma risk. Then, we conducted log-rank test, Kaplan-Meier analysis and Cox regression model to evaluate the relationship of PPARD and PPARG polymorphisms with glioma prognosis. We found PPARD polymorphisms (rs2016520, rs67056409, rs1053049) were significantly associated with glioma risk in multiple models $(P<0.05)$. Stratified analysis showed rs2016520, rs67056409, rs1053049 of PPARD significantly decreased risk of glioma in the subgroup of age $>40$ and astrocytoma $(P<0.05)$. For male, PPARD rs1053049 had a strong relationship with glioma risk in allele $(P=0.041)$, dominant $(P=0.040)$ and additive $(P=0.040)$ models. The effect of PPARG rs 2920503 on glioma risk was related to glioma grade $(P<0.05)$. MDR showed that a seven-locus model was the best polymorphisms interaction pattern. Moreover, surgery and chemotherapy had strongly impact on overall survival and progression free survival of glioma patients. Our findings suggested that PPARD and PPARG polymorphisms were associated with glioma risk and prognosis in the Chinese Han population, and further studies are need to confirm our results.

Glioma is the most common type of malignant brain tumors in the central nervous system (CNS), accounting for approximately $80 \%$ of primary brain tumors ${ }^{1}$. The incidence of brain cancer is the highest in European (5.5/100,000 persons), North America (5.3/100,000 persons), Australia (5.3/100,000 persons), Western Asia (5.2/100,000 persons) and Northern Africa (5.0/100,000 persons) ${ }^{2}$. In China, there were 1,016,000 newly diagnosed cases of brain and CNS tumor in $2015^{3}$. Glioma occurs varied in age, sex, race, histologic type and geographic characteristics ${ }^{4}$. And, glioma has poor overall survival (OS), with less than 5 year survival of patients after diagnosis ${ }^{5}$. The etiology of glioma is multifactorial, which is the results of environmental exposure and genetic factors ${ }^{4}$. Single nucleotide polymorphism (SNP) is the most studied mutations involved in genetic predisposition of glioma. Recently, increasing studies are focused on the role of Peroxisome proliferator-activated receptors (PPARs) polymorphisms on cancer.

PPARs is a subfamily of nuclear receptor transcription factors and consists three isoforms (PPAR $\alpha$, PPAR $\delta$ and PPAR $\gamma$ ). PPARD encodes PPAR $\delta$, a nuclear hormone receptor that implicated in varieties of biological processes, including epidermal cell proliferation, migration, lipid and glucose metabolism ${ }^{6-8}$. PPARD is highly expressed in brain, heart, skeletal muscle, adipose tissue and pancreatic islets ${ }^{9}$. In mice, $P P A R D$ agonists increase leptin secretion and improve type 2 diabetes ${ }^{10,11}$. The overexpression of $P P A R D$ was observed in various human cancers, such as colorectal, pancreatic and lung cancer ${ }^{12-15}$. Previous studies revealed that $P P A R D$ polymorphisms were associated with lipid levels, metabolic traits, obesity and risk of coronary heart diseases (CHD) and cancers $^{16-19}$. PPARD rs2016520 is located in the $5^{\prime}$-untranslated region of exon, which has been widely studied in

\footnotetext{
${ }^{1}$ Department of Anesthesia, The Second Affiliated Hospital of Xi'an Jiaotong University, Xi'an, Shaanxi, 710004, China. ${ }^{2}$ Department of Pediatric Surgery, The Second Affiliated Hospital of Xi'an Jiaotong University, Xi'an, Shaanxi, 710004, China. *email:ygao@mail.xjtu.edu.cn
} 
multiple physiological and pathological process ${ }^{20-23}$. However, little is known on the relationship of PPARD polymorphisms with glioma risk and prognosis.

PPARG is located in human chromosome 3 p25 and encodes a nuclear receptor (PPAR $\gamma$ ) activated by fatty acid metabolites or synthetic medicines ${ }^{24-26}$. PPARG is mainly expressed in suprabasal keratinocytes, adipocyte tissue, vascular endothelial cells, macrophage cells and smooth muscle cells ${ }^{27,28}$. PPARG regulates adipocyte differentiation and controls genes expression involved in lipid and glucose homeostasis ${ }^{29}$. And, PPARG has anti-inflammatory effect by restraining the production of inflammatory mediators ${ }^{30}$. It has been reported that PPARG is implicated in the pathology of obesity, diabetes, atherosclerosis and cancer. Wang et al. indicated that PPARG could arrest cell growth in human oral cancer ${ }^{31}$. Fan et al. pointed that anti- PPARG therapy is a potential strategy to improve endocrine-resistant breast cancer ${ }^{32}$. Nevertheless, the role of $P P A R G$ in glioma has not been elucidated.

Therefore, we conducted a case-control study to investigate the association of PPARD and PPARG polymorphisms (rs2016520, rs67056409, rs1053049, rs2206030, rs2920503, rs4073770 and rs1151988) with glioma risk and prognosis in the Chinese Han population.

\section{Methods}

Study population. This study consisted of 568 glioma patients and 509 healthy controls, recruited from the Second Affiliated Hospital of Xi'an Jiaotong University, Shaanxi Province, China. All glioma patients were newly diagnosed and histologically confirmed according to the World Health Organization (WHO) classification ${ }^{33}$. The exclusion criteria of glioma patients are as follows: (1) patients have history of cancer or CNS diseases; (2) patients are under 18 years old. The controls were healthy individuals without history of cancer or serious diseases who randomly enrolled from the same hospital. We obtained demographic and clinical information of study population from medical records and follow-up. This study was performed in accordance with the Declaration of Helsinki, and it was approved by the ethics committee of the Second Affiliated Hospital of Xi'an Jiaotong University. Informed consents were required form all participants before this study.

SNP selection and genotyping. Combined previously studies, we selected four SNPs of PPARD (rs2016520, rs67056409, rs1053049 and rs2206030) and three SNPs of PPARG (rs2920503, rs4073770 and rs1151988), with minor allele frequencies (MAF) greater than 5\% in the HapMap Chinese Han Beijing population. We extracted DNA from peripheral blood samples using the blood DNA kit (GoldMag Co. Ltd., Xi'an, China). SNP genotyping was performed in the Agena MassARRAY system (Agena, San Diego, CA, USA). Primers for polymerase chain reaction (PCR) amplification and extension were designed by the Agena MassARRAY Assay Design 3.0 Software (San Diego, CA USA). PCR primers of selected SNPs were listed in Supplemental Table 1. In addition, we used Agena Typer 4.0 Software (San Diego, CA, USA) to manage and analyze data.

Statistical analysis. We conducted all statistical analysis using Microsoft Excel and SPSS version 21.0 software (SPSS, Chicago, IL, USA). Student's $t$-test and chi-square test were used to compare the differences in age and sex between glioma patients and healthy controls. The Hardy-Weinberg equilibrium (HWE) was checked for controls with Fisher's exact test. We assessed the association of PPARD and PPARG polymorphisms with glioma risk by calculating odd ratios (OR) and 95\% confidence intervals (CI) using logistic regression. Multifactor dimensionality reduction (MDR, version 3.0.2) was used to analyze SNP-SNP interactions on glioma risk. Then, we plotted patient survival curves by the Kaplan-Meier method and log-rank test. The association of PPARD and PPARG polymorphisms with OS and progression free survival (PFS) of glioma patients was evaluated by calculating hazard ratios (HR) and 95\%CI using univariate and multivariate analysis. In multivariable survival analysis, we assessed the associations of PPARD and PPARG polymorphisms with glioma prognosis adjusted by age, sex, WHO grade, surgery, radiotherapy and chemotherapy. All tests were two-sided, and $P<0.05$ was regarded as statistical significance. Additionally, our results were adjusted for multiple comparison using false discovery rate (FDR) correction.

\section{Results}

Characteristics of study population. The characteristics of 568 glioma patients and 509 healthy controls were presented in Table 1. The mean ages of the cases and controls were $39.68 \pm 16.96$ and $41.32 \pm 15.69$ years old, respectively. No significant variation in age or sex was found between the two groups (age: $P=0.102$, sex: $P=1.000)$. Among glioma patients, 438 (77\%) people were astrocytoma. According to WHO grading standards, 35 (6\%) patients were grade I, 320 (56\%) patients were grade II, and others are in high-grade glioma (III + IV). In addition, surgery method, radiotherapy and chemotherapy of patients were shown in Table 1.

Association of PPARD and PPARG polymorphisms with glioma risk. In Table 2, PPARD and $P P A R G$ polymorphisms were accord with HWE in controls $(P>0.05)$.

HaploReg (https://pubs.broadinstitute.org/mammals/haploreg/haploreg.php) predicted that PPARD and PPARG polymorphisms were related to the regulation of SiPhy cons, Promoter histone marks, Enhancer histone marks, DNAse, Motifs changed, GRASP QTL hits, NHGRI/EBI GWAS hits, Selected eQTL. After adjustment for age and sex, PPARD polymorphisms (rs2016520, rs67056409 and rs1053049) were significantly associated with glioma risk $(P<0.05)$. Rs2016520 and rs 1053049 of $P P A R D$ had a decreased glioma risk in allele (rs2016520: $\mathrm{OR}=0.82,95 \% \mathrm{CI}=0.68-0.99, P=0.041 ; \mathrm{rs} 1053049: \mathrm{OR}=0.78,95 \% \mathrm{CI}=0.64-0.95, P=0.012)$, dominant $(\mathrm{rs} 2016520: \mathrm{OR}=0.78,95 \% \mathrm{CI}=0.62-1.00, P=0.047 ; \mathrm{rs} 1053049: \mathrm{OR}=0.75,95 \% \mathrm{CI}=0.59-0.96, P=0.020)$ and additive $(\mathrm{rs} 2016520: \mathrm{OR}=0.81,95 \% \mathrm{CI}=0.67-0.99, P=0.037 ; \mathrm{rs} 1053049: \mathrm{OR}=0.78,95 \% \mathrm{CI}=0.64-0.95$, $P=0.012$ ) models. We found that the allele distribution of rs67056409 were significantly different between cases and controls $(P=0.046)$, and subjects had lower risk of glioma in additive model $(\mathrm{OR}=0.82,95 \% \mathrm{CI}=0.67-$ $0.99, P=0.041)$. There were no significant association between glioma risk and other genetic polymorphisms (rs2206030, rs2920503, rs4073770 and rs1151988). However, FDR analysis revealed that the significant associations between genetic polymorphisms and glioma risk were not reliable. 


\begin{tabular}{|l|l|l|l|}
\hline Characteristics & $\begin{array}{l}\text { Glioma patients } \\
(\text { N=568) }\end{array}$ & $\begin{array}{l}\text { Healthy controls } \\
(\text { N=509) }\end{array}$ & $\boldsymbol{P}$ \\
\hline Age & $39.68 \pm 16.96$ & $41.32 \pm 15.69$ & 0.102 \\
\hline$>40$ & $296(52 \%)$ & $241(47 \%)$ & \\
\hline$\leq 40$ & $272(48 \%)$ & $268(53 \%)$ & \\
\hline Sex & & & 1.000 \\
\hline Male & $313(55 \%)$ & $280(55 \%)$ & \\
\hline Female & $255(45 \%)$ & $229(45 \%)$ & \\
\hline Astrocytoma & & & \\
\hline Yes & $438(77 \%)$ & & \\
\hline No & $130(23 \%)$ & & \\
\hline WHO grade & $35(6 \%)$ & & \\
\hline I & $320(56 \%)$ & & \\
\hline II & $149(26 \%)$ & & \\
\hline III & $64(12 \%)$ & & \\
\hline IV & & & \\
\hline Surgery & $181(32 \%)$ & & \\
\hline STR \& NTR & $387(68 \%)$ & & \\
\hline GTR & & & \\
\hline Radiotherapy & $59(10 \%)$ & & \\
\hline No & $154(27 \%)$ & & \\
\hline Conformable radiotherapy & $355(63 \%)$ & & \\
\hline Gamma knife & \multicolumn{5}{|l}{} & \\
\hline Chemotherapy & $182(32 \%)$ & & \\
\hline No & & & \\
\hline Yes-temodar & & & \\
\hline Yes-not temodar & & & \\
\hline
\end{tabular}

Table 1. Comparison of glioma patients and controls by characteristics. WHO, World Health Organization; STR, sub-total resection; NTR, near-total resection; GTR, gross-total resection.

We further did stratification analysis of PPARD and PPARG polymorphisms with glioma risk (Tables 3 and 4). For the subjects older than 40 years old, rs2016520, rs67056409 and rs1053049 of PPARD significantly decreased risk of glioma in multiple models $(P<0.05)$. Rs 1053049 had a strong relationship with decreased risk of glioma in the subgroup of male (allele: $\mathrm{OR}=0.76,95 \% \mathrm{CI}=0.59-0.99, P=0.041$; dominant: $\mathrm{OR}=0.71,95 \% \mathrm{CI}=0.51-0.98$, $P=0.040$; additive: $\mathrm{OR}=0.76,95 \% \mathrm{CI}=0.59-0.99, P=0.040)$. Then, we divided glioma patients to astrocytoma and others, we found that rs2016520, rs67056409 and rs1053049 of PPARD were significantly associated astrocytoma risk compared with other glioma $(P<0.05)$. We also explored the effect of WHO grade on the relationship of genetic polymorphisms with glioma risk. The results showed PPARG rs2920503 was strongly related to higher risk of high-grade glioma $(\mathrm{III}+\mathrm{IV})$ in co-dominant $(\mathrm{OR}=2.04,95 \% \mathrm{CI}=1.13-3.68, P=0.018)$ and recessive $(\mathrm{OR}=2.03$, $95 \% \mathrm{CI}=1.15-3.57, P=0.014)$ models. After FDR correction, the protective effects of rs2016520, rs67056409 and rs1053049 on glioma risk were still significant among the individuals older than 40 years old (FDR- $P<0.05)$.

MDR analysis. We used MDR analysis to assess the impact of the interaction among seven SNPs. The results obtained from MDR analysis for one- to seven- locus modes were presented in Table 5. A seven-locus model including polymorphisms of PPARD (rs2016520, rs67056409, rs1053049 and rs2206030) and PPARG (rs2920503, rs4073770 and rs1151988) was the best model of SNP-SNP interaction for glioma risk (cross-validation consistency $=10 / 10$, accuracy $=0.660$, sensitivity $=0.751$, specificity $=0.570, P<0.001$ ).

Clinical factors and glioma prognosis. After obtained follow-up data of glioma patients, we investigated the impact of clinical factors on glioma prognosis (OS and PFS). As shown in Table 6, surgery and chemotherapy had significant correlations with OS and PFS of glioma $(P<0.05$, FDR- $P<0.05)$. The prognosis of patients had gross-total resection (GTR) was better than those had sub-total resection (STR) or near-total resection (NTR) (OS: Log-rank $P=1.54 \mathrm{E}-07, \mathrm{HR}=0.63,95 \% \mathrm{CI}=0.53-0.77, P=1.88 \mathrm{E}-06$, FDR- $P=1.32 \mathrm{E}-05$; PFS: Log-rank $P=1.91 \mathrm{E}-09, \mathrm{HR}=0.59,95 \% \mathrm{CI}=0.49-0.71, P=6.78 \mathrm{E}-08$, FDR- $P=4.75 \mathrm{E}-07)$. Additionally, glioma patients who undergone chemotherapy lived longer than those not (OS: Log-rank $P=1.38 \mathrm{E}-05, \mathrm{HR}=0.69$, $95 \% \mathrm{CI}=0.58-0.83, P=7.47 \mathrm{E}-05$, FDR- $P=0.000261$; PFS: Log-rank $P=0.005, \mathrm{HR}=0.79,95 \% \mathrm{CI}=0.66-0.95$, $P=0.011$, FDR- $P=0.039$ ). There were no significantly associations between other clinical factors (sex, age, WHO grade and radiotherapy) and glioma prognosis $(P>0.05)$.

Association of PPARD and PPARG polymorphisms with glioma prognosis. Then, we assessed the association of PPARD (rs2016520, rs67056409, rs1053049 and rs2206030) and PPARG (rs2920503, rs4073770 and rs1151988) polymorphisms with glioma prognosis. In Supplemental Table 2, univariate analysis did not show a 


\begin{tabular}{|c|c|c|c|c|c|c|c|c|c|c|c|c|c|}
\hline \multirow[b]{2}{*}{ Gene } & \multirow[b]{2}{*}{ SNP } & \multirow[b]{2}{*}{ Chr: position } & \multirow[b]{2}{*}{ HaploReg v4.1 } & \multirow[b]{2}{*}{ Group } & \multicolumn{3}{|c|}{ Genotype } & \multicolumn{2}{|c|}{ Allele frequency } & \multirow[b]{2}{*}{ Model } & \multirow[b]{2}{*}{ OR(95\%CI) } & \multirow[b]{2}{*}{$P$} & \multirow[b]{2}{*}{ FDR- -} \\
\hline & & & & & AA & $\mathbf{A B}$ & BB & $\begin{array}{l}\text { MAF } \\
\text { (A) }\end{array}$ & HWE- $P$ & & & & \\
\hline \multirow[t]{6}{*}{ PPARD } & rs 2016520 & 6: 35378778 & \multirow{6}{*}{$\begin{array}{l}\text { SiPhy cons, } \\
\text { Promoter histone } \\
\text { marks, Enhancer } \\
\text { histone marks, } \\
\text { DNAse, Motifs } \\
\text { changed, GRASP } \\
\text { QTL hits, } \\
\text { Selected eQTL }\end{array}$} & case & 31 & 217 & 317 & 0.247 & & Allele & $0.82(0.68-0.99)$ & 0.041 & 0.214 \\
\hline & & & & control & 37 & 217 & 255 & 0.286 & 0.385 & Co-dominant & $0.67(0.40-1.11)$ & 0.123 & 0.323 \\
\hline & & & & & & & & & & & $0.80(0.62-1.03)$ & 0.085 & 0.275 \\
\hline & & & & & & & & & & Dominant & $0.78(0.62-1.00)$ & 0.047 & 0.214 \\
\hline & & & & & & & & & & Recessive & $0.74(0.45-1.21)$ & 0.231 & 0.404 \\
\hline & & & & & & & & & & Additive & $0.81(0.67-0.99)$ & 0.037 & 0.214 \\
\hline \multirow[t]{6}{*}{ PPARD } & rs67056409 & 6: 35383699 & \multirow{6}{*}{$\begin{array}{l}\text { Promoter histone } \\
\text { marks, Enhancer } \\
\text { histone marks, } \\
\text { DNAse, Motifs } \\
\text { changed, } \\
\text { Selected eQTL }\end{array}$} & case & 32 & 226 & 310 & 0.255 & & Allele & $0.82(0.68-1.00)$ & 0.046 & 0.214 \\
\hline & & & & control & 40 & 219 & 250 & 0.294 & 0.455 & Co-dominant & $0.64(0.39-1.06)$ & 0.081 & 0.275 \\
\hline & & & & & & & & & & & $0.83(0.65-1.07)$ & \begin{tabular}{|l|}
0.147 \\
\end{tabular} & 0.323 \\
\hline & & & & & & & & & & Dominant & $0.80(0.63-1.02)$ & 0.072 & 0.275 \\
\hline & & & & & & & & & & Recessive & $0.70(0.43-1.13)$ & 0.146 & 0.323 \\
\hline & & & & & & & & & & Additive & $0.82(0.67-0.99)$ & \begin{tabular}{|l|}
0.041 \\
\end{tabular} & 0.214 \\
\hline \multirow[t]{6}{*}{ PPARD } & rs1053049 & 6: 35395618 & \multirow{6}{*}{$\begin{array}{l}\text { DNAse, Motifs } \\
\text { changed, GRASP } \\
\text { QTL hits, } \\
\text { Selected eQTL }\end{array}$} & case & 30 & 203 & 334 & 0.232 & & Allele & $0.78(0.64-0.95)$ & 0.012 & 0.214 \\
\hline & & & & control & 39 & 206 & 264 & 0.279 & 1.000 & Co-dominant & $0.60(0.37-1.00)$ & 0.051 & 0.214 \\
\hline & & & & & & & & & & & $0.78(0.60-1.00)$ & 0.051 & 0.214 \\
\hline & & & & & & & & & & Dominant & $0.75(0.59-0.96)$ & \begin{tabular}{|l|}
0.020 \\
\end{tabular} & 0.214 \\
\hline & & & & & & & & & & Recessive & $0.67(0.41-1.10)$ & 0.114 & 0.323 \\
\hline & & & & & & & & & & Additive & $0.78(0.64-0.95)$ & 0.012 & 0.214 \\
\hline \multirow[t]{6}{*}{ PPARD } & rs 2206030 & 6:35404354 & \multirow{6}{*}{$\begin{array}{l}\text { Enhancer } \\
\text { histone marks, } \\
\text { Motifs changed, } \\
\text { NHGRI/EBI } \\
\text { GWAS hits, } \\
\text { Selected eQTL }\end{array}$} & case & 126 & 291 & 151 & 0.478 & & Allele & $1.08(0.91-1.28)$ & 0.371 & 0.546 \\
\hline & & & & control & 106 & 255 & 148 & 0.459 & 0.929 & Co-dominant & $1.17(0.83-1.65)$ & 0.382 & 0.546 \\
\hline & & & & & & & & & & & $1.12(0.84-1.48)$ & 0.436 & 0.573 \\
\hline & & & & & & & & & & Dominant & $1.13(0.87-1.48)$ & 0.361 & 0.546 \\
\hline & & & & & & & & & & Recessive & $1.08(0.81-1.45)$ & 0.587 & 0.685 \\
\hline & & & & & & & & & & Additive & $1.08(0.91-1.29)$ & 0.365 & 0.546 \\
\hline \multirow[t]{6}{*}{ PPARG } & rs 2920503 & 3: 12324230 & & case & 55 & 233 & 280 & 0.302 & & Allele & $1.00(0.83-1.20)$ & 0.985 & 0.986 \\
\hline & & & & control & 43 & 221 & 245 & 0.302 & 0.529 & Co-dominant & $1.12(0.72-1.73)$ & 0.612 & 0.695 \\
\hline & & & Metifs choned & & & & & & & & $0.92(0.72-1.19)$ & 0.529 & 0.635 \\
\hline & & & Motifs changed & & & & & & & Dominant & $0.95(0.75-1.21)$ & 0.702 & 0.776 \\
\hline & & & & & & & & & & Recessive & $1.16(0.76-1.77)$ & 0.482 & 0.595 \\
\hline & & & & & & & & & & Additive & $1.00(0.83-1.20)$ & 0.986 & 0.986 \\
\hline PPARG & rs4073770 & $3: 12368233$ & & case & 60 & 265 & 243 & 0.339 & & Allele & $0.99(0.83-1.19)$ & 0.924 & 0.972 \\
\hline & & & & control & 69 & 209 & 231 & 0.341 & 0.061 & Co-dominant & $0.83(0.56-1.22)$ & 0.339 & 0.546 \\
\hline & & & $\begin{array}{l}\text { Enhancer } \\
\text { histone marks, }\end{array}$ & & & & & & & & $1.21(0.93-1.56)$ & 0.152 & 0.323 \\
\hline & & & Motifs changed, & & & & & & & Dominant & $1.11(0.87-1.41)$ & 0.390 & 0.546 \\
\hline & & & & & & & & & & Recessive & $0.75(0.52-1.09)$ & 0.132 & 0.323 \\
\hline & & & & & & & & & & Additive & $0.99(0.83-1.18)$ & 0.925 & 0.971 \\
\hline PPARG & rs1151988 & 3: 12511512 & & case & 7 & 133 & 428 & 0.129 & & Allele & $0.84(0.66-1.07)$ & 0.162 & 0.324 \\
\hline & & & Enhancer histone & control & 9 & 135 & 365 & 0.150 & 0.488 & Co-dominant & $0.66(0.24-1.80)$ & 0.418 & 0.566 \\
\hline & & & marks, Motifs & & & & & & & & $0.84(0.64-1.11)$ & 0.217 & 0.396 \\
\hline & & & $\begin{array}{l}\text { changed, GRASP } \\
\text { QTL hits, }\end{array}$ & & & & & & & Dominant & $0.83(0.63-1.09)$ & 0.175 & 0.334 \\
\hline & & & Selected eQTL & & & & & & & Recessive & $0.69(0.26-1.87)$ & 0.470 & 0.595 \\
\hline & & & & & & & & & & Additive & $0.83(0.65-1.07)$ & 0.154 & 0.323 \\
\hline
\end{tabular}

Table 2. Association of PPARD and PPARG polymorphisms with glioma risk. SNP, single nucleotide polymorphism; MAF, minor allele frequency; HWE, Hardy-Weinberg equilibrium; OR, odds ratio; CI, confidence interval; FDR, false discovery rate. Bold values indicate statistical significance $(P<0.05)$.

strong relationship of PPARD and PPARG polymorphisms with glioma prognosis $(P>0.05)$. Moreover, we did not observe significantly association of $P P A R D$ and $P P A R G$ polymorphisms with OS and PFS of glioma patients $(P>0.05$, Supplemental Table 3).

\section{Discussion}

In this case-control study, we examined the association of $P P A R D$ and $P P A R G$ polymorphisms with glioma risk and prognosis in the Chinese Han population. After FDR correction, we found that $P P A R D$ polymorphisms were significantly associated with glioma risk, and the effects were dependent on age $(P<0.05$, FDR- $P<0.05)$. Moreover, surgery method and chemotherapy had strongly effects on glioma prognosis (Log-rank $P<0.05, P<0.05$, FDR- $P<0.05$ ).

PPARs are involved in the regulation of metabolic homeostasis, whose activity are controlled by fatty acid ligands ${ }^{34}$. After activation, PPARs heterodimerize with retinoid X receptors (RXRs) to affect the expression of 


\begin{tabular}{|c|c|c|c|c|c|c|c|c|c|c|c|c|c|c|}
\hline \multirow[b]{3}{*}{ Gene } & \multirow[b]{3}{*}{ SNP } & \multirow[b]{3}{*}{ Model } & \multicolumn{6}{|l|}{ Age } & \multicolumn{6}{|l|}{ Sex } \\
\hline & & & \multicolumn{3}{|l|}{$>40$} & \multicolumn{3}{|l|}{$\leq 40$} & \multicolumn{3}{|l|}{ Male } & \multicolumn{3}{|l|}{ Female } \\
\hline & & & OR(95\%CI) & $P$ & FDR-P & $\mathrm{OR}(95 \% \mathrm{CI})$ & $P$ & FDR-P & OR(95\%CI) & $P$ & FDR-P & OR(95\%CI) & $P$ & FDR- $P$ \\
\hline \multirow[t]{6}{*}{ PPARD } & rs2016520 & Allele & 0.66(0.50-0.88) & 0.004 & 0.034 & $1.01(0.77-1.31)$ & 0.964 & 0.970 & 0.82(0.64-1.06) & 0.130 & 0.455 & $0.82(0.61-1.09)$ & 0.170 & 0.476 \\
\hline & & Co-dominant & $0.48(0.22-1.06)$ & 0.070 & 0.173 & $0.90(0.45-1.79)$ & 0.765 & 0.970 & $0.78(0.4-1.51)$ & 0.460 & 0.855 & $0.54(0.24-1.19)$ & 0.124 & 0.476 \\
\hline & & & $0.61(0.43-0.88)$ & 0.008 & 0.047 & $1.08(0.75-1.55)$ & 0.675 & 0.970 & $0.74(0.53-1.04)$ & 0.084 & 0.370 & $0.89(0.61-1.29)$ & 0.526 & 0.757 \\
\hline & & Dominant & $0.60(0.42-0.84)$ & 0.004 & 0.034 & $1.05(0.74-1.49)$ & 0.774 & 0.970 & 0.75(0.54-1.03) & 0.079 & 0.370 & $0.83(0.58-1.19)$ & 0.312 & 0.624 \\
\hline & & Recessive & $0.59(0.27-1.28)$ & 0.179 & 0.376 & $0.87(0.45-1.69)$ & 0.678 & 0.970 & \begin{tabular}{|l|}
$0.89(0.47-1.70)$ \\
\end{tabular} & 0.730 & 0.902 & $0.56(0.26-1.23)$ & 0.149 & 0.476 \\
\hline & & Additive & $0.65(0.48-0.87)$ & 0.004 & 0.034 & $1.01(0.76-1.33)$ & 0.955 & 0.970 & $0.81(0.62-1.06)$ & 0.119 & 0.454 & $0.81(0.60-1.09)$ & 0.164 & 0.476 \\
\hline \multirow[t]{6}{*}{ PPARD } & rs67056409 & Allele & $0.65(0.49-0.86)$ & 0.003 & 0.034 & $1.03(0.80-1.34)$ & 0.811 & 0.970 & \begin{tabular}{|l|}
$0.80(0.62-1.03)$ \\
\end{tabular} & 0.088 & 0.370 & $0.85(0.64-1.14)$ & 0.276 & 0.580 \\
\hline & & Co-dominant & $0.40(0.19-0.85)$ & 0.017 & 0.071 & $0.99(0.5-1.96)$ & 0.969 & 0.970 & \begin{tabular}{|l|}
$0.73(0.38-1.41)$ \\
\end{tabular} & 0.347 & 0.855 & $0.54(0.25-1.16)$ & 0.112 & 0.476 \\
\hline & & & $0.67(0.46-0.96)$ & 0.027 & 0.095 & 1.12(0.78-1.6) & 0.544 & 0.970 & $0.73(0.52-1.02)$ & 0.069 & 0.370 & $0.97(0.67-1.41)$ & 0.883 & 0.883 \\
\hline & & Dominant & $0.62(0.44-0.88)$ & 0.007 & 0.047 & $1.10(0.78-1.55)$ & 0.596 & 0.970 & \begin{tabular}{|l|}
$0.73(0.53-1.01)$ \\
\end{tabular} & 0.058 & 0.370 & $0.90(0.63-1.29)$ & 0.562 & 0.757 \\
\hline & & Recessive & $0.47(0.22-0.98)$ & 0.045 & 0.135 & $0.93(0.48-1.82)$ & 0.842 & 0.970 & $0.84(0.45-1.59)$ & 0.595 & 0.855 & $0.54(0.26-1.15)$ & 0.111 & 0.476 \\
\hline & & Additive & 0.65(0.49-0.86) & 0.003 & 0.034 & $1.05(0.79-1.39)$ & 0.732 & 0.970 & 0.79(0.61-1.03) & 0.082 & 0.370 & $0.85(0.63-1.13)$ & 0.264 & 0.580 \\
\hline \multirow[t]{6}{*}{ PPARD } & rs 1053049 & Allele & $0.68(0.51-0.91)$ & 0.009 & 0.047 & $0.89(0.68-1.17)$ & 0.407 & 0.970 & $0.76(0.59-0.99)$ & 0.041 & 0.370 & $0.80(0.60-1.08)$ & 0.143 & 0.476 \\
\hline & & Co-dominant & $0.41(0.18-0.93)$ & 0.033 & 0.107 & $0.80(0.41-1.57)$ & 0.518 & 0.970 & $0.63(0.33-1.21)$ & 0.163 & 0.527 & $0.56(0.25-1.25)$ & 0.159 & 0.476 \\
\hline & & & $0.71(0.50-1.03)$ & 0.069 & 0.173 & $0.88(0.61-1.26)$ & 0.473 & 0.970 & $0.73(0.52-1.02)$ & 0.065 & 0.370 & $0.85(0.58-1.23)$ & 0.385 & 0.703 \\
\hline & & Dominant & $0.67(0.47-0.95)$ & 0.024 & 0.092 & $0.86(0.61-1.22)$ & 0.406 & 0.970 & $0.71(0.51-0.98)$ & 0.040 & 0.370 & $0.80(0.56-1.15)$ & 0.232 & 0.573 \\
\hline & & Recessive & $0.47(0.21-1.05)$ & 0.065 & 0.173 & $0.85(0.44-1.63)$ & 0.625 & 0.970 & 0.72(0.39-1.36) & 0.315 & 0.855 & $0.60(0.27-1.32)$ & 0.204 & 0.536 \\
\hline & & Additive & $0.68(0.51-0.91)$ & 0.010 & 0.047 & $0.89(0.67-1.17)$ & 0.389 & 0.970 & $0.76(0.59-0.99)$ & 0.040 & 0.370 & $0.80(0.59-1.08)$ & 0.142 & 0.476 \\
\hline \multirow[t]{6}{*}{ PPARD } & rs2206030 & Allele & $1.04(0.82-1.33)$ & 0.726 & 0.828 & $1.10(0.86-1.40)$ & 0.446 & 0.970 & $1.09(0.87-1.37)$ & 0.460 & 0.855 & $1.07(0.83-1.38)$ & 0.605 & 0.757 \\
\hline & & Co-dominant & $1.11(0.68-1.81)$ & 0.683 & 0.820 & $1.25(0.75-2.07)$ & 0.391 & 0.970 & $1.19(0.74-1.91)$ & 0.471 & 0.855 & $1.14(0.69-1.89)$ & 0.613 & 0.757 \\
\hline & & & $1.29(0.85-1.96)$ & 0.232 & 0.424 & $0.97(0.65-1.44)$ & 0.866 & 0.970 & 1.13(0.78-1.65) & 0.516 & 0.855 & $1.10(0.72-1.69)$ & 0.659 & 0.762 \\
\hline & & Dominant & $1.23(0.83-1.83)$ & 0.304 & 0.532 & $1.04(0.71-1.51)$ & 0.848 & 0.970 & $1.15(0.80-1.64)$ & 0.448 & 0.855 & $1.11(0.74-1.67)$ & 0.602 & 0.757 \\
\hline & & Recessive & $0.93(0.62-1.40)$ & 0.735 & 0.828 & $1.27(0.82-1.98)$ & 0.280 & 0.970 & $1.10(0.73-1.64)$ & 0.653 & 0.885 & $1.07(0.70-1.63)$ & 0.753 & 0.791 \\
\hline & & Additive & $1.06(0.83-1.35)$ & 0.665 & 0.820 & $1.10(0.86-1.41)$ & 0.459 & 0.970 & \begin{tabular}{|l|}
$1.09(0.87-1.38)$ \\
\end{tabular} & 0.450 & 0.855 & \begin{tabular}{|l|}
$1.07(0.83-1.38)$ \\
\end{tabular} & 0.606 & 0.757 \\
\hline \multirow[t]{6}{*}{ PPARG } & rs2920503 & Allele & $1.00(0.77-1.30)$ & 0.996 & 0.996 & $1.01(0.78-1.30)$ & 0.970 & 0.970 & $1.05(0.82-1.34)$ & 0.717 & 0.902 & $0.95(0.72-1.25)$ & 0.705 & 0.762 \\
\hline & & Co-dominant & $1.15(0.61-2.17)$ & 0.671 & 0.820 & $1.07(0.57-1.98)$ & 0.840 & 0.970 & $1.18(0.65-2.14)$ & 0.583 & 0.855 & $1.05(0.55-2.00)$ & 0.878 & 0.883 \\
\hline & & & $0.91(0.64-1.30)$ & 0.604 & 0.794 & $0.91(0.63-1.31)$ & 0.612 & 0.970 & \begin{tabular}{|l|}
$0.99(0.71-1.39)$ \\
\end{tabular} & 0.971 & 0.971 & 0.84(0.58-1.23) & 0.368 & 0.703 \\
\hline & & Dominant & $0.95(0.67-1.33)$ & 0.749 & 0.828 & $0.94(0.66-1.33)$ & 0.710 & 0.970 & $1.02(0.74-1.41)$ & 0.891 & 0.959 & $0.88(0.61-1.25)$ & 0.469 & 0.757 \\
\hline & & Recessive & $1.20(0.65-2.22)$ & 0.561 & 0.794 & $1.11(0.61-2.02)$ & 0.722 & 0.970 & $1.18(0.67-2.09)$ & 0.561 & 0.855 & $1.14(0.61-2.11)$ & 0.685 & 0.762 \\
\hline & & Additive & $1.00(0.77-1.31)$ & 0.996 & 0.996 & $0.98(0.75-1.28)$ & 0.900 & 0.970 & $1.05(0.82-1.35)$ & 0.715 & 0.902 & $0.95(0.72-1.25)$ & 0.708 & 0.762 \\
\hline \multirow[t]{6}{*}{ PPARG } & rs 4073770 & Allele & $0.94(0.73-1.21)$ & 0.605 & 0.794 & $1.05(0.82-1.35)$ & 0.689 & 0.970 & $1.07(0.84-1.37)$ & 0.573 & 0.855 & $0.90(0.69-1.18)$ & 0.449 & 0.757 \\
\hline & & Co-dominant & 0.79(0.45-1.40) & 0.414 & 0.669 & $0.86(0.50-1.50)$ & 0.599 & 0.970 & 1.05(0.62-1.80) & 0.848 & 0.960 & $0.63(0.35-1.12)$ & 0.118 & 0.476 \\
\hline & & & $1.02(0.71-1.46)$ & 0.930 & 0.976 & $1.40(0.97-2.04)$ & 0.076 & 0.970 & $1.17(0.83-1.65)$ & 0.371 & 0.855 & $1.25(0.85-1.83)$ & 0.256 & 0.580 \\
\hline & & Dominant & $0.97(0.68-1.36)$ & 0.843 & 0.908 & $1.25(0.88-1.78)$ & 0.206 & 0.970 & $1.14(0.83-1.58)$ & 0.415 & 0.855 & $1.07(0.75-1.54)$ & 0.704 & 0.762 \\
\hline & & Recessive & 0.78(0.46-1.34) & 0.372 & 0.625 & $0.73(0.43-1.23)$ & 0.237 & 0.970 & 0.98(0.59-1.62) & 0.925 & 0.971 & $0.56(0.33-0.97)$ & 0.038 & 0.476 \\
\hline & & Additive & $0.93(0.72-1.20)$ & 0.569 & 0.794 & $1.04(0.81-1.34)$ & 0.736 & 0.970 & $1.07(0.84-1.36)$ & 0.575 & 0.855 & $0.91(0.70-1.18)$ & 0.455 & 0.757 \\
\hline \multirow[t]{6}{*}{ PPARG } & rs1151988 & Allele & $0.78(0.54-1.12)$ & 0.170 & 0.376 & $0.91(0.66-1.28)$ & 0.599 & 0.970 & 0.96(0.69-1.33) & 0.799 & 0.932 & $0.72(0.50-1.03)$ & 0.074 & 0.476 \\
\hline & & Co-dominant & 0.52(0.09-3.20) & 0.482 & 0.750 & $0.80(0.23-2.75)$ & 0.723 & 0.970 & 0.71(0.19-2.68) & 0.610 & 0.855 & $0.61(0.13-2.76)$ & 0.520 & 0.757 \\
\hline & & & $0.78(0.52-1.17)$ & 0.224 & 0.424 & $0.91(0.61-1.35)$ & 0.645 & 0.970 & $0.99(0.68-1.45)$ & 0.969 & 0.971 & $0.69(0.46-1.04)$ & 0.076 & 0.476 \\
\hline & & Dominant & $0.77(0.52-1.14)$ & 0.191 & 0.382 & $0.9(0.62-1.33)$ & 0.602 & 0.970 & 0.97(0.67-1.41) & 0.885 & 0.960 & $0.68(0.46-1.02)$ & 0.065 & 0.476 \\
\hline & & Recessive & 0.55(0.09-3.38) & 0.523 & 0.785 & $0.82(0.24-2.81)$ & 0.751 & 0.970 & 0.71(0.19-2.68) & 0.611 & 0.855 & $0.67(0.15-3.03)$ & 0.602 & 0.757 \\
\hline & & Additive & \begin{tabular}{|l|}
$0.77(0.53-1.12)$ \\
\end{tabular} & 0.171 & 0.376 & $0.91(0.64-1.28)$ & 0.577 & 0.970 & $0.96(0.68-1.34)$ & 0.793 & 0.932 & $0.71(0.49-1.03)$ & 0.068 & 0.476 \\
\hline
\end{tabular}

Table 3. Association of $P P A R D$ and $P P A R G$ polymorphisms with glioma risk stratified by age and sex. SNP, single nucleotide polymorphism; MAF, minor allele frequency; HWE, Hardy-Weinberg equilibrium; OR, odds ratio; CI, confidence interval; FDR, false discovery rate. Bold values indicate statistical significance $(P<0.05)$.

downstream genes. It is reported that PPARs might had a functional crosstalk concerning the control of their expression $^{35}$. Previous studies on the role of PPARs signaling in cancer mainly based on the availability of PPARs agonists and antagonists ${ }^{36}$. In brain tumor stem cells, PPAR $\gamma$ agonists inhibit cell growth and induce cell cycle arrest $^{37}$. In mice, expression of PPAR $\delta$ is related to prognosis and metastatic ability of breast cancer cells $\mathrm{s}^{38}$. Polymorphisms of PPARD and PPARG are associated with risk and prognosis of many diseases, including cardiovascular disease, diabetes, brain diseases, medulloblastoma and other cancers ${ }^{39-41}$. In our study, we firstly observed that PPARD polymorphisms (rs2016520, rs67056409 and rs1053049) were significantly associated with glioma risk. Similar association has been reported in colorectal cancer ${ }^{39}$. It suggests that PPARD polymorphisms could be involved in the susceptibility of glioma development. And, stratified analysis showed the effects 


\begin{tabular}{|c|c|c|c|c|c|c|c|c|}
\hline \multirow[b]{2}{*}{ Gene } & \multirow[b]{2}{*}{ SNP } & \multirow[b]{2}{*}{ Model } & \multicolumn{3}{|c|}{ Astrocytoma VS. Other glioma } & \multicolumn{3}{|c|}{$\begin{array}{l}\text { WHO grade } \\
\text { (III + IV VS. I + II) }\end{array}$} \\
\hline & & & $\mathrm{OR}(95 \% \mathrm{CI})$ & $P$ & FDR- $P$ & OR $(95 \% \mathrm{CI})$ & $P$ & FDR- $P$ \\
\hline \multirow[t]{6}{*}{ PPARD } & rs2016520 & Allele & $0.79(0.64-0.97)$ & 0.025 & 0.224 & $0.97(0.73-1.28)$ & 0.810 & 0.989 \\
\hline & & Co-dominant & $0.60(0.34-1.05)$ & 0.072 & 0.236 & $1.08(0.50-2.33)$ & 0.844 & 0.989 \\
\hline & & & $0.80(0.61-1.05)$ & 0.103 & 0.254 & $0.98(0.68-1.41)$ & 0.923 & 0.992 \\
\hline & & Dominant & $0.77(0.60-1.00)$ & 0.048 & 0.224 & $0.99(0.70-1.41)$ & 0.973 & 0.992 \\
\hline & & Recessive & $0.66(0.38-1.14)$ & 0.135 & 0.315 & $1.09(0.51-2.32)$ & 0.826 & 0.989 \\
\hline & & Additive & $0.79(0.64-0.97)$ & 0.027 & 0.224 & $1.01(0.76-1.35)$ & 0.956 & 0.992 \\
\hline \multirow[t]{6}{*}{ PPARD } & rs67056409 & Allele & $0.80(0.65-0.98)$ & 0.033 & 0.224 & $0.93(0.70-1.22)$ & 0.598 & 0.989 \\
\hline & & Co-dominant & $0.58(0.34-1.01)$ & 0.054 & 0.227 & $0.80(0.36-1.77)$ & 0.584 & 0.989 \\
\hline & & & $0.84(0.64-1.10)$ & 0.198 & 0.362 & $1.03(0.72-1.48)$ & 0.863 & 0.989 \\
\hline & & Dominant & $0.80(0.62-1.03)$ & 0.089 & 0.239 & $1.00(0.71-1.42)$ & 0.992 & 0.992 \\
\hline & & Recessive & $0.63(0.37-1.08)$ & 0.091 & 0.239 & $0.79(0.36-1.72)$ & 0.554 & 0.989 \\
\hline & & Additive & $0.80(0.65-0.99)$ & 0.039 & 0.224 & $0.97(0.73-1.29)$ & 0.828 & 0.989 \\
\hline \multirow[t]{6}{*}{ PPARD } & rs1053049 & Allele & $0.76(0.61-0.93)$ & 0.009 & 0.224 & $0.92(0.69-1.23)$ & 0.581 & 0.989 \\
\hline & & Co-dominant & $0.56(0.32-0.98)$ & 0.043 & 0.224 & $0.87(0.39-1.95)$ & 0.744 & 0.989 \\
\hline & & & $0.77(0.59-1.02)$ & 0.064 & 0.236 & $0.97(0.67-1.40)$ & 0.871 & 0.989 \\
\hline & & Dominant & $0.74(0.57-0.96)$ & 0.024 & 0.224 & $0.96(0.67-1.36)$ & 0.810 & 0.989 \\
\hline & & Recessive & $0.62(0.36-1.08)$ & 0.091 & 0.239 & $0.89(0.40-1.95)$ & 0.762 & 0.989 \\
\hline & & Additive & $0.76(0.62-0.94)$ & 0.012 & 0.224 & $0.95(0.71-1.28)$ & 0.754 & 0.989 \\
\hline \multirow[t]{6}{*}{ PPARD } & rs2206030 & Allele & $1.09(0.91-1.31)$ & 0.342 & 0.497 & $1.08(0.85-1.38)$ & 0.510 & 0.989 \\
\hline & & Co-dominant & $1.18(0.82-1.70)$ & 0.376 & 0.497 & $1.14(0.69-1.88)$ & 0.615 & 0.989 \\
\hline & & & $1.09(0.81-1.48)$ & 0.572 & 0.632 & $1.38(0.91-2.10)$ & 0.131 & 0.523 \\
\hline & & Dominant & $1.12(0.84-1.49)$ & 0.447 & 0.539 & $1.30(0.88-1.94)$ & 0.192 & 0.620 \\
\hline & & Recessive & $1.12(0.82-1.52)$ & 0.489 & 0.555 & $0.92(0.61-1.39)$ & 0.684 & 0.989 \\
\hline & & Additive & $1.09(0.91-1.30)$ & 0.372 & 0.497 & $1.08(0.84-1.38)$ & 0.560 & 0.989 \\
\hline \multirow[t]{6}{*}{ PPARG } & rs2920503 & Allele & $0.91(0.75-1.12)$ & 0.379 & 0.497 & $1.27(0.98-1.64)$ & 0.074 & 0.499 \\
\hline & & Co-dominant & $0.92(0.57-1.49)$ & 0.744 & 0.801 & $2.04(1.13-3.68)$ & 0.018 & 0.378 \\
\hline & & & $0.85(0.65-1.11)$ & 0.235 & 0.386 & $1.02(0.70-1.47)$ & 0.933 & 0.992 \\
\hline & & Dominant & $0.86(0.67-1.11)$ & 0.255 & 0.397 & $1.17(0.83-1.65)$ & 0.377 & 0.989 \\
\hline & & Recessive & $0.99(0.63-1.58)$ & 0.982 & 0.982 & $2.03(1.15-3.57)$ & 0.014 & 0.378 \\
\hline & & Additive & $0.91(0.75-1.12)$ & 0.370 & 0.497 & $1.27(0.98-1.64)$ & 0.074 & 0.499 \\
\hline \multirow[t]{6}{*}{ PPARG } & rs 4073770 & Allele & $1.02(0.85-1.24)$ & 0.818 & 0.838 & $0.80(0.62-1.03)$ & 0.083 & 0.499 \\
\hline & & Co-dominant & $0.86(0.56-1.31)$ & 0.480 & 0.555 & $0.70(0.38-1.27)$ & 0.240 & 0.672 \\
\hline & & & $1.28(0.98-1.69)$ & 0.073 & 0.236 & $0.73(0.51-1.06)$ & 0.095 & 0.499 \\
\hline & & Dominant & $1.18(0.91-1.53)$ & 0.211 & 0.369 & $0.73(0.51-1.03)$ & 0.071 & 0.499 \\
\hline & & Recessive & $0.76(0.51-1.13)$ & 0.170 & 0.340 & $0.82(0.46-1.45)$ & 0.489 & 0.989 \\
\hline & & Additive & $1.03(0.85-1.24)$ & 0.799 & 0.838 & $0.80(0.61-1.04)$ & 0.092 & 0.499 \\
\hline \multirow[t]{6}{*}{ PPARG } & rs1151988 & Allele & $0.83(0.64-1.08)$ & 0.160 & 0.340 & $1.25(0.88-1.78)$ & 0.209 & 0.627 \\
\hline & & Co-dominant & $0.62(0.21-1.88)$ & 0.403 & 0.513 & $1.54(0.34-7.05)$ & 0.580 & 0.989 \\
\hline & & & $0.84(0.62-1.13)$ & 0.239 & 0.386 & $1.34(0.90-2.00)$ & 0.154 & 0.539 \\
\hline & & Dominant & $0.82(0.61-1.10)$ & 0.191 & 0.362 & $1.35(0.91-2.00)$ & 0.137 & 0.523 \\
\hline & & Recessive & $0.65(0.22-1.97)$ & 0.449 & 0.539 & $1.43(0.31-6.52)$ & 0.646 & 0.989 \\
\hline & & Additive & $0.83(0.63-1.08)$ & 0.164 & 0.340 & $1.32(0.92-1.90)$ & 0.137 & 0.523 \\
\hline
\end{tabular}

Table 4. Association of $P P A R D$ and $P P A R G$ polymorphisms with glioma risk stratified by pathological classification and WHO grade. SNP, single nucleotide polymorphism; MAF, minor allele frequency; HWE, Hardy-Weinberg equilibrium; OR, odds ratio; CI, confidence interval; FDR, false discovery rate. Bold values indicate statistical significance $(P<0.05)$.

of PPARD polymorphisms on glioma risk were age-dependent. It provides a scientific basis on individualized treatment of glioma. The effects of $P P A R D$ polymorphisms on glioma risk might related to SiPhy cons, Promoter histone marks, Enhancer histone marks, DNAse, Motifs changed, GRASP QTL hits, NHGRI/EBI GWAS hits, Selected eQTL. However, our results should be confirmed in further studies, including next-generation technology, PCR, western-blot analysis, etc.

Glioma is likely to have unfavorable prognosis caused by rapid proliferation and diffuse brain invasion. Despite surgery, chemotherapy and radiotherapy treatments improve, the prognosis of glioma remains poor ${ }^{42}$. Recent studies reported that some lipophilic molecules have antiproliferation and/or differentiation effects on glioma cells, and PPARs mediated some activities of these processes ${ }^{43}$. PPAR $\gamma$ has been observed in transformed neural cells of human and PPAR $\gamma$ agonist interferes with glioma growth and malignancy ${ }^{43-45}$. In this study, we 


\begin{tabular}{|l|l|l|l|l|l|l|l|l|}
\hline Model & $\begin{array}{l}\text { Bal. Acc. CV } \\
\text { Training }\end{array}$ & $\begin{array}{l}\text { Bal. Acc. } \\
\text { CV Testing }\end{array}$ & $\begin{array}{l}\text { CV } \\
\text { Consistency }\end{array}$ & Accuracy & Sensitivity & Specificity & OR(95\%CI) & P \\
\hline rs1053049 & 0.538 & 0.513 & $8 / 10$ & 0.537 & 0.593 & 0.481 & $1.35(1.06-1.74)$ & $\mathbf{0 . 0 1 7}$ \\
\hline rs1053049, rs2206030 & 0.554 & 0.512 & $5 / 10$ & 0.550 & 0.646 & 0.454 & $1.52(1.18-1.95)$ & $\mathbf{0 . 0 0 1}$ \\
\hline $\begin{array}{l}\text { rs2920503, rs1053049, } \\
\text { rs2206030 }\end{array}$ & 0.573 & 0.471 & $2 / 10$ & 0.566 & 0.554 & 0.578 & $1.70(1.33-2.18)$ & $<\mathbf{0 . 0 0 1}$ \\
\hline $\begin{array}{l}\text { rs2920503, rs4073770, } \\
\text { rs2016520, rs2206030 }\end{array}$ & 0.602 & 0.500 & $7 / 10$ & 0.597 & 0.646 & 0.548 & $2.22(1.72-2.85)$ & $<\mathbf{0 . 0 0 1}$ \\
\hline $\begin{array}{l}\text { rs2920503, rs4073770, } \\
\text { r1151983, rs2016520, } \\
\text { rs2206030 }\end{array}$ & 0.630 & 0.500 & $5 / 10$ & 0.624 & 0.601 & 0.646 & $2.76(2.14-3.55)$ & $<\mathbf{0 . 0 0 1}$ \\
\hline $\begin{array}{l}\text { rs2920503, rs4073770, } \\
\text { rs1151988, rs67056409, } \\
\text { rs1053049, rs2206030 }\end{array}$ & 0.658 & 0.506 & $10 / 10$ & 0.650 & 0.751 & 0.550 & $3.68(2.82-4.80)$ & $<\mathbf{0 . 0 0 1}$ \\
\hline $\begin{array}{l}\text { rs2920503, rs4073770, } \\
\text { rs1151988, rs2016520, } \\
\text { rs67056409, rs1053049, } \\
\text { rs2206030 }\end{array}$ & 0.668 & 0.4951 & $10 / 10$ & 0.660 & 0.751 & 0.570 & $3.98(3.05-5.20)$ & $<\mathbf{0 . 0 0 1}$ \\
\hline
\end{tabular}

Table 5. MDR analysis of SNP-SNP interaction. MDR, multifactor dimensionality reduction; SNP, single nucleotide polymorphism; $\mathrm{CV}$, cross-validation; $\mathrm{OR}$, odds ratio; $\mathrm{CI}$, confidence interval. Bold values indicate statistical significance $(P<0.05)$.

\begin{tabular}{|c|c|c|c|c|c|c|c|c|c|c|c|c|c|}
\hline \multirow{2}{*}{\multicolumn{2}{|c|}{ Variables }} & \multirow[b]{2}{*}{ Total } & \multirow[b]{2}{*}{ Event } & \multicolumn{4}{|l|}{ OS } & \multicolumn{6}{|l|}{ PFS } \\
\hline & & & & $\begin{array}{l}\text { Log-rank } \\
P\end{array}$ & \begin{tabular}{|l|} 
SR \\
(1-/3-year)
\end{tabular} & HR $(95 \% \mathrm{CI})$ & $P$ & FDR- $P$ & \begin{tabular}{|l|} 
Log- \\
rank $P$
\end{tabular} & \begin{tabular}{|l|} 
SR \\
(1-/3-year)
\end{tabular} & HR $(95 \% \mathrm{CI})$ & $P$ & FDR- $P$ \\
\hline \multirow{2}{*}{ Sex } & Male & 313 & 278 & \multirow{2}{*}{0.379} & $0.328 / 0.082$ & \multirow{2}{*}{$1.08(0.92-1.28)$} & \multirow{2}{*}{0.420} & \multirow{2}{*}{0.490} & \multirow{2}{*}{0.268} & $0.200 / 0.096$ & \multirow{2}{*}{$1.09(0.92-1.30)$} & \multirow{2}{*}{0.321} & \multirow{2}{*}{0.321} \\
\hline & Female & 255 & 229 & & $0.310 / 0.094$ & & & & & $0.155 / 0.089$ & & & \\
\hline \multirow{2}{*}{ Age } & $<40$ & 250 & 215 & \multirow{2}{*}{0.074} & $0.355 / 0.117$ & \multirow{2}{*}{$1.16(0.97-1.38)$} & \multirow{2}{*}{0.101} & \multirow{2}{*}{0.235} & \multirow{2}{*}{0.064} & $0.206 / 0.119$ & \multirow{2}{*}{$1.16(0.97-1.38)$} & \multirow{2}{*}{0.097} & \multirow{2}{*}{0.136} \\
\hline & $\geq 40$ & 317 & 291 & & $0.290 / 0.067$ & & & & & $0.159 / 0.072$ & & & \\
\hline \multirow{2}{*}{ WHO grade } & $\mathrm{I}+\mathrm{II}$ & 355 & 311 & \multirow{2}{*}{0.121} & $0.328 / 0.108$ & \multirow{2}{*}{$1.14(0.95-1.36)$} & \multirow{2}{*}{0.155} & \multirow{2}{*}{0.271} & \multirow{2}{*}{0.096} & $0.192 / 0.108$ & \multirow{2}{*}{$1.15(0.96-1.37)$} & \multirow{2}{*}{0.136} & \multirow{2}{*}{0.159} \\
\hline & $\mathrm{III}+\mathrm{IV}$ & 213 & 196 & & $0.305 / 0.064$ & & & & & $0.158 / 0.067$ & & & \\
\hline \multirow{2}{*}{ Surgery } & $\begin{array}{l}\text { NTR \& } \\
\text { STR }\end{array}$ & 181 & 178 & \multirow{2}{*}{$1.54 \mathrm{E}-07$} & $0.204 /-$ & \multirow{2}{*}{$0.63(0.53-0.77)$} & $1.88 \mathrm{E}-06$ & $1.32 \mathrm{E}-05$ & $1.91 \mathrm{E}-09$ & $0.016 /-$ & $0.59(0.49-0.71)$ & $6.78 \mathrm{E}-08$ & 4.75E-07 \\
\hline & GTR & 387 & 329 & & $0.374 / 0.123$ & & & & & $0.254 / 0.126$ & & & \\
\hline Radiotherapy & No & 59 & 48 & & $0.441 /-$ & & & & & $0.200 /-$ & & & \\
\hline Conformal radi & otherapy & 154 & 126 & 0.438 & $0.250 / 0.152$ & $1.08(0.78-1.51)$ & 0.636 & 0.636 & 0.118 & $0.217 / 0.154$ & $1.37(0.97-1.94)$ & 0.070 & 0.136 \\
\hline & $\begin{array}{l}\text { Gamma } \\
\text { knife }\end{array}$ & 355 & 333 & & $0.330 / 0.055$ & $1.17(0.87-1.59)$ & 0.303 & 0.424 & & $0.163 / 0.058$ & $1.31(0.96-1.80)$ & 0.090 & 0.136 \\
\hline & No & 337 & 315 & $1.38358 \mathrm{E}-$ & $0.276 / 0.029$ & $060-0-0003)$ & 7.46734E- & & 0005 & $0.164 / 0.057$ & & 0011 & \\
\hline Cnemotnerapy & Yes & 231 & 192 & & \begin{tabular}{|l|}
$0.384 / 0.151$ \\
\end{tabular} & $0.69(0.58-0.83)$ & & 0.000261 & 0.005 & $0.205 / 0.155$ & $0.19(0.66-0.95)$ & 0.011 & 0.039 \\
\hline
\end{tabular}

Table 6. The impact of clinical factors on glioma patient OS and PFS. OS, overall survival; PFS, progression free survival; SR, survival rate; HR, hazard ratio; CI, confidence interval; WHO, World Health Organization; STR, sub-total resection; NTR, near-total resection; GTR, gross-total resection; FDR, false discovery rate. Bold values indicate statistical significance $(P<0.05)$.

firstly confirmed the effects of surgery method and chemotherapy on prognosis of glioma patients. Then, we explored the association of PPARD and PPARG polymorphisms with OS and PFS of glioma patients. No significant associations were observed by univariate and multivariate analysis. It demonstrated that PPARD and PPARG polymorphisms might not contribute the prognosis of glioma.

There are some limitations in the present study. First, we selected and genotyped several polymorphisms of PPARD and PPARG, more genetic polymorphisms should be studied in the future. Second, we could not evaluate more factors on the association of genetic polymorphisms and glioma risk due to the limited sample size and information. Third, the molecular mechanisms of PPARD and PPARG on glioma risk and prognosis are not elucidated in our study.

\section{Conclusion}

In conclusion, we found genetic polymorphisms of $P P A R D$ were associated with glioma risk in the Chinese Han population, which suggests the role of PPARD in the carcinogenesis of glioma.

It provided information on exploring the mechanism and targeted therapy of glioma, it also promotes the development of precision medicine on glioma. Further studies in larger samples with more ethnic groups are needed to validate our results and explore the mechanism of PPARD and PPARG in glioma. 
Received: 23 October 2019; Accepted: 4 February 2020;

Published online: 20 March 2020

\section{References}

1. Ostrom, Q. T. et al. CBTRUS statistical report: primary brain and other central nervous system tumors diagnosed in the United States in 2012-2016. Neuro-oncology 21, v1-v100 (2019).

2. Ferlay, J. et al. GLOBOCAN 2012 v1. 0, cancer incidence and mortality worldwide: IARC CancerBase No. 11. Lyon, France: International agency for research on cancer 2016 (2013).

3. Chen, W. et al. Cancer statistics in China, 2015. CA: a cancer J. clinicians 66, 115-132 (2016).

4. Ostrom, Q. T., Gittleman, H., Stetson, L., Virk, S. M. \& Barnholtzsloan, J. S. Epidemiology of gliomas. Cancer Treat. Res. 163, 1-14 (2015).

5. Stupp, R. et al. Radiotherapy plus concomitant and adjuvant temozolomide for glioblastoma. N. Engl. J. Med. 352, 987-996 (2005).

6. Tan, N. S. et al. The nuclear hormone receptor peroxisome proliferator-activated receptor $\beta / \delta$ potentiates cell chemotactism, polarization, and migration. Mol. Cell. Biol. 27, 7161-7175 (2007).

7. Lee, C.-H. et al. PPAR $\delta$ regulates glucose metabolism and insulin sensitivity. Proc. Natl Acad. Sci. 103, 3444-3449 (2006).

8. Schmuth, M. et al. Peroxisome proliferator-activated receptor (PPAR)- $\beta / \delta$ stimulates differentiation and lipid accumulation in keratinocytes. J. Investig. Dermatology 122, 971-983 (2004).

9. Berger, J. \& Moller, D. The mechanisms of action of ppars. Annu Rev Med. (2002).

10. Luquet, S. et al. Peroxisome proliferator-activated receptor $\delta$ controls muscle development and oxidative capability. FASEB J. 17, 2299-2301 (2003).

11. Wang, Y. X. et al. Regulation of Muscle Fiber Type and Running Endurance by PPAR $\delta$. PLoS Biol. 2, e294 (2004).

12. He, T.-C., Chan, T. A., Vogelstein, B. \& Kinzler, K. W. PPAR $\delta$ is an APC-regulated target of nonsteroidal anti-inflammatory drugs. Cell 99, 335-345 (1999).

13. Gupta, R. A. et al. Prostacyclin-mediated activation of peroxisome proliferator-activated receptor $\delta$ in colorectal cancer. Proc. Natl Acad. Sci. 97, 13275-13280 (2000).

14. Abdollahi, A. et al. Transcriptional network governing the angiogenic switch in human pancreatic cancer. Proc. Natl Acad. Sci. 104, 12890-12895 (2007).

15. Pedchenko, T. V., Gonzalez, A. L., Wang, D., DuBois, R. N. \& Massion, P. P. Peroxisome proliferator-activated receptor $\beta / \delta$ expression and activation in lung cancer. Am. J. respiratory Cell Mol. Biol. 39, 689-696 (2008).

16. Seedorf, U. \& Aberle, J. Emerging roles of PPAR $\delta$ in metabolism. Biochimica et. Biophysica Acta-Molecular Cell Biol. Lipids 1771, $1125-1131$ (2007).

17. Tang, L., Lü, Q., Cao, H., Yang, Q. \& Tong, N. PPARD rs2016520 polymorphism is associated with metabolic traits in a large population of Chinese adults. Gene 585, 191-195 (2016).

18. Luo, W. et al. A population association study of PPAR $\delta$ gene rs 2016520 and rs 9794 polymorphisms and haplotypes with body mass index and waist circumference in a Chinese population. Ann. Hum. Biol. 43, 67-72 (2016).

19. Panza, A. et al. Peroxisome proliferator-activated receptor $\gamma$-mediated induction of microRNA-145 opposes tumor phenotype in colorectal cancer. Biochim. Biophys. Acta 1843, 1225-1236 (2014).

20. Jin-Fang, S. et al. PPARD rs2016520 polymorphism affects repaglinide response in Chinese Han patients with type?2 diabetes mellitus. Clin. Exp. Pharmacology Physiol. 42, 27-32 (2015).

21. Hautala, A. J. et al. Peroxisome proliferator-activated receptor- $\delta$ polymorphisms are associated with physical performance and plasma lipids: the HERITAGE Family Study. Am. J. Physiol. Heart Circ. Physiol 292, H2498-2505 (2007).

22. Yan, Z. et al. PPARdelta $+294 \mathrm{~T} / \mathrm{C}$ gene polymorphism related to plasma lipid, obesity and left ventricular hypertrophy in subjects with metabolic syndrome. Zhonghua Xin Xue Guan Bing. Za Zhi 33, 529-533 (2005).

23. Skogsberg, J. et al. Peroxisome proliferator activated receptor delta genotype in relation to cardiovascular risk factors and risk of coronary heart disease in hypercholesterolaemic men. J. Intern. Med. 254, 597-604 (2003).

24. Desvergne, Ba, Michalik, L. \& Wahli, W. Be fit or be sick: peroxisome proliferator-activated receptors are down the road. Mol. Endocrinol. 18, 1321-1332 (2004).

25. Straus, D. S. \& Glass, C. K. Anti-inflammatory actions of PPAR ligands: new insights on cellular and molecular mechanisms. Trends immunology 28, 551-558 (2007)

26. Varga, T., Czimmerer, Z. \& Nagy, L. PPARs are a unique set of fatty acid regulated transcription factors controlling both lipid metabolism and inflammation. Biochimica et. Biophysica Acta-Molecular Basis Dis. 1812, 1007-1022 (2011).

27. Neve, B. P., Fruchart, J.-C. \& Staels, B. Role of the peroxisome proliferator-activated receptors (PPAR) in atherosclerosis. Biochemical pharmacology 60, 1245-1250 (2000).

28. Icre, G., Wahli, W. \& Michalik, L. In Journal of Investigative Dermatology Symposium Proceedings. 30-35 (Elsevier).

29. Calle, E. E. \& Kaaks, R. Overweight, obesity and cancer: epidemiological evidence and proposed mechanisms. Nat. Rev. Cancer 4, $579(2004)$

30. Hutter, S., Knabl, J., Andergassen, U. \& Jeschke, U. The role of PPARs in placental immunology: a systematic review of the literature. PPAR research 2013 (2013).

31. Wang, H.-Y. et al. Rosiglitazone elevates sensitization of drug-resistant oral epidermoid carcinoma cells to vincristine by G2/Mphase arrest, independent of PPAR- $\gamma$ pathway. Biomedicine Pharmacotherapy 83, 349-361 (2016).

32. Fan, P., Abderrahman, B., Chai, T. S., Yerrum, S. \& Jordan, V. C. Targeting Peroxisome Proliferator-Activated Receptor $\gamma$ to Increase Estrogen-Induced Apoptosis in Estrogen-Deprived Breast Cancer Cells. Mol. cancer therapeutics 17, 2732-2745 (2018).

33. Guo, X. et al. Impact of ANXA5 polymorphisms on glioma risk and patient prognosis. J. neuro-oncology 142, 11-26 (2019).

34. Chang, W. H. \& Lai, A. G. Pan-cancer mutational landscape of the PPAR pathway reveals universal patterns of dysregulated metabolism and interactions with tumor immunity and hypoxia. bioRxiv, 563676 (2019).

35. Ding, Y. et al. Gene-Gene Interaction between PPAR $\delta$ and PPAR $\gamma$ Is Associated with Abdominal Obesity in a Chinese Population. J. Genet. genomics 39, 625-631 (2012).

36. Laganà, A. et al. Pleiotropic actions of peroxisome proliferator-activated receptors (PPARs) in dysregulated metabolic homeostasis, inflammation and cancer: Current evidence and future perspectives. Int. J. Mol. Sci. 17, 999 (2016).

37. Chearwae, W. \& Bright, J. PPAR $\gamma$ agonists inhibit growth and expansion of CD133+ brain tumour stem cells. Br. J. Cancer 99, 2044 (2008).

38. Wang, X. et al. PPAR-delta promotes survival of breast cancer cells in harsh metabolic conditions. Oncogenesis 5, e232 (2016).

39. Rosales-Reynoso, M. et al. Protective role of +294 T/C (rs2016520) polymorphism of PPARD in Mexican patients with colorectal cancer. Genet. Mol. Res 16 (2017).

40. Huang, Y. et al. PPARD rs2016520 polymorphism and circulating lipid levels connect with brain diseases in Han Chinese and suggest sex-dependent effects. Biomedicine Pharmacotherapy 70, 7-11 (2015).

41. Ye, H. et al. Positive association between PPARD rs2016520 polymorphism and coronary heart disease in a Han Chinese population. Genet. Mol. Res. 14, 6350-6359 (2015).

42. Coras, R. et al. The peroxisome proliferator-activated receptor- $\gamma$ agonist troglitazone inhibits transforming growth factor- $\beta$-mediated glioma cell migration and brain invasion. Mol. Cancer Therapeutics 6, 1745-1754 (2007). 
43. Benedetti, E. et al. Biomolecular characterization of human glioblastoma cells in primary cultures: differentiating and antiangiogenic effects of natural and synthetic PPAR $\gamma$ agonists. J. Cell. Physiol. 217, 93-102 (2008).

44. Benedetti, E., Galzio, R., D’Angelo, B., Ceru, M. P. \& Cimini, A. PPARs in human neuroepithelial tumors: PPAR ligands as anticancer therapies for the most common human neuroepithelial tumors. PPAR research 2010 (2010).

45. Grommes, C. et al. Inhibition of in vivo glioma growth and invasion by peroxisome proliferator-activated receptor $\gamma$ agonist treatment. Mol. pharmacology 70, 1524-1533 (2006).

\section{Acknowledgements}

We sincerely thank all participants in this study. This study was supported by Shaanxi province natural science basic research program (2017JM3018).

\section{Author contributions}

Xiaoying Ding and Ya Gao designed this study, Xinsheng Han and Haozheng Yuan collected samples, Yong Zhang wrote the manuscript, Ya Gao revised the draft and supervised this study.

\section{Competing interests}

The authors declare no competing interests.

\section{Additional information}

Supplementary information is available for this paper at https://doi.org/10.1038/s41598-020-60996-2.

Correspondence and requests for materials should be addressed to Y.G.

Reprints and permissions information is available at www.nature.com/reprints.

Publisher's note Springer Nature remains neutral with regard to jurisdictional claims in published maps and institutional affiliations.

(c) (i) Open Access This article is licensed under a Creative Commons Attribution 4.0 International

License, which permits use, sharing, adaptation, distribution and reproduction in any medium or format, as long as you give appropriate credit to the original author(s) and the source, provide a link to the Creative Commons license, and indicate if changes were made. The images or other third party material in this article are included in the article's Creative Commons license, unless indicated otherwise in a credit line to the material. If material is not included in the article's Creative Commons license and your intended use is not permitted by statutory regulation or exceeds the permitted use, you will need to obtain permission directly from the copyright holder. To view a copy of this license, visit http://creativecommons.org/licenses/by/4.0/.

(c) The Author(s) 2020 